\title{
THE USE OF SPECTRAL AND TEXTURAL FEATURES IN CROP TYPE MAPPING USING SENTINEL-2A IMAGES: A CASE STUDY, ÇUKUROVA REGION, TURKEY
}

\author{
A. Tuzcu Kokal ${ }^{1 *}$, A. F. Sunar ${ }^{1}$, A. Dervisoglu ${ }^{1}$, S. Berberoglu ${ }^{2}$ \\ ${ }^{1}$ ITU, Civil Engineering Faculty, 34469 Maslak Istanbul, Turkey - (tuzcuay, fsunar, adervisoglu)@itu.edu.tr \\ ${ }^{2}$ Çukurova University, Agriculture Faculty, Adana, Turkey - suha@cu.edu.tr
}

Commission III, WG III/1

KEY WORDS: Crop mapping, Sentinel-2A, GLCM, Object-based classification, Spectral and textural analysis

\begin{abstract}
:
Turkey has favorable agricultural conditions (i.e. fertile soils, climate and rainfall) and can grow almost any type of crop in many regions, making it one of the leading sectors of the economy. For sustainable agriculture management, all factors affecting the agricultural products should be analyzed on a spatial-temporal basis. Therefore, nowadays space technologies such as remote sensing are important tools in providing an accurate mapping of the agricultural fields with timely monitoring and higher repetition frequency and accuracy. In this study, object based classification method was applied to 2017 Sentinel 2 Level 2A satellite image in order to map crop types in the Adana, Çukurova region in Turkey. Support Vector Machine (SVM) was used as a classifier. Texture information were incorporated to spectral wavebands of Sentinel-2 image, to increase the classification accuracy. In this context, all of the textural features of Gray-Level Co-occurrence Matrix (GLCM) were tested and Entropy, Standard deviation, and Mean textural features were found to be the most suitable among them. Multi-spectral and textural features were used as an input separately and/or in combination to evaluate the potential of texture in differentiating crop types and the accuracy of output thematic maps. As a result, with the addition of textural features, it was observed that the Overall Accuracy and Kappa coefficient increased by $7 \%$ and $8 \%$, respectively.
\end{abstract}

\section{INTRODUCTION}

Monitoring cropland has a profound impact on environmental planning, food security, and crop yield estimation. According to the estimates of the United Nations, the world population has now reached approximately 7.7 billion (United Nations, 2019). Due to the growing population, the need for agricultural production, which is the main component of the food supply, has become very important in the last few decades. As in many countries, agriculture, parallel to the continuous increase in the share of industry and services, it is one of the leading sectors of Turkey's economy. Since fertile soil, favorable climate, and rainfall conditions allow almost all types of crop products to be grown in many parts of Turkey, the total agricultural cultivated area is about 23 million ha in 2019 according to the Turkish Statistical Institute (TURKSTAT, 2020). Today, remote sensing technology that maximizes the use of cultivated areas fosters the development of sustainable agriculture and helps farmers better manage resources such as water, pesticides, and fertilizers. Therefore, satellite images are considered a potentially important tool for studying vegetation from local to global.

In order to extract information from satellite images, different image processing steps can be applied. Among these steps, the most popular one is thematic classification. Types of classification methods can be supervised or unsupervised, as well as pixel-based, parcel-based or object-based. Although pixelbased classification is the most appropriate approach to classifying low- and medium-resolution satellite images, objectbased classification, in which objects are classified instead of pixels, has become increasingly popular with the increase in spatial resolutions. One of the important reasons for its popularity is that object-based methods made it possible to analyze the shape and textural variation of the aggregated sets of pixels, as well as spectral properties (Blaschke, 2010).

In general, the input dataset used in image classification consists of spectral information (i.e. spectral reflectance properties), which is an effective tool to capture the physical and chemical properties of earth features such as vegetation. In addition to spectral information, textural information containing useful information needed to identify the features is used by deriving spatial variations in a satellite image. Image texture defines the image content through the spatial distribution pattern and it is commonly evaluated as variation and repetition of grey levels or local patterns. A widely used statistical method that uses the spatial relationship of pixels to extract texture information in an image is the Gray-Level Co-occurrence Matrix (GLCM) (Haralick et al., 1973). Specifically, GLCM is defined as tabulating the frequency of occurrence according to a different combination of pixel intensity values in an image (Zainal et.al., 2013).

There are several studies devoted to crop type mapping that use pixel-based or object-based classification with different features such as NDVI, GLCM textures from time series or single-date remote sensing image (Akkartal et al., 2004, Avci and Sunar, 2015; Goodin et.al., 2015; Kim and Yeom, 2014; Ok et.al., 2012). It was also reported that the texture features contributed to classification accuracy by providing additional information (Trias-Sanz et al., 2008; Blaschke, 2010; Peña-Barragán et al., 2011).

\footnotetext{
* Corresponding author
} 
In this study, the effect of spectral and textural features on crop mapping accuracy was analyzed using the 2017 Sentinel 2 Level $2 \mathrm{~A}$ image in the Çukurova region, which has high agricultural productivity. For this purpose, crop type mapping was performed with an object-based classification using advanced supervised machine-learning algorithms such as Support Vector Machine $(\mathrm{SVM})$.

\section{DATA AND METHODOLOGY}

\subsection{Study Area and Data Used}

The study area is located on the southeast Mediterranean coast of Turkey, called Cukurova Delta. This area stretches from the coastal strip to the land for about $17-30 \mathrm{~km}$ and has different landscapes ranging from the alluvial plain called Çukurova to the smooth undulating terrain and mountains to river deltas. The plain represents the sedimentary sediment formed by the three main rivers of the region, Ceyhan, Seyhan and Berdan, flowing from the Taurus Mountains to the Mediterranean. The main land use is agriculture (38\% i.e. 540,000 ha arable land), as the region has fertile alluvial soils and mild climate (Alphan and Yilmaz, 2005). In this region, an area of $10 \times 10 \mathrm{~km}^{2}$ containing different crop species was selected for this study (Figure 1).

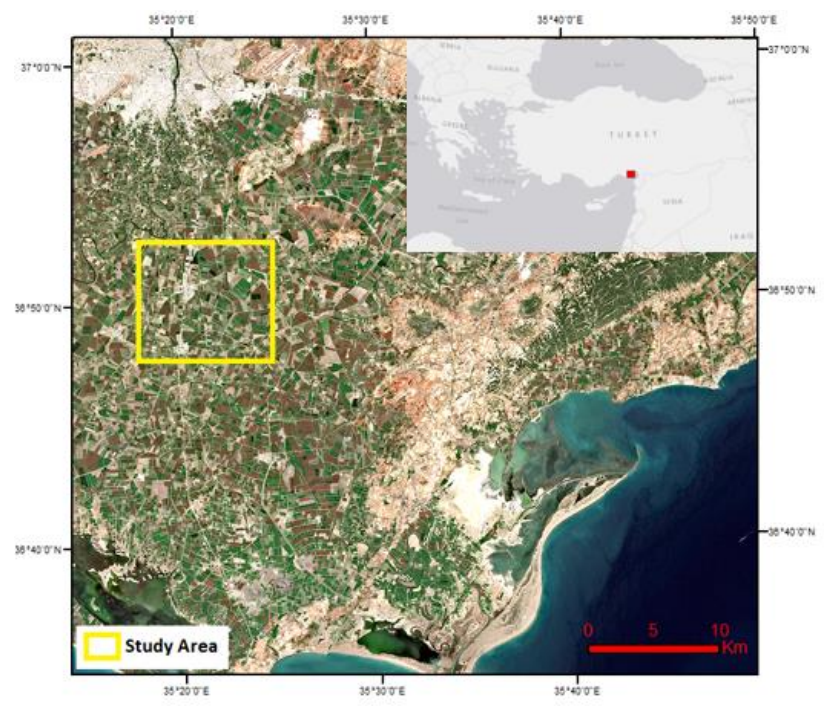

Figure 1. Satellite image map of the study area.

Ground truth data were collected through field survey conducted on August 13, 2017 and was used as reference data in training and validation processes. Since different numbers of samples were collected for various crops in the field survey, corn (20 parcels) and soybean (5 parcels), which have the highest number of samples among different crop types, were mainly considered in this study. The cultivation timetable for major crops in the region is given in Figure 2.

In the analysis, cloudless Sentinel 2 Level 2A Sub-Atmosphere (BOA) reflection image data obtained on July 30, 2017 were selected to be as simultaneous as possible with the ground truth data. As input spectral features, all spectral bands of Sentinel 2A satellite image except coastal aerosol, water vapor, SWIR-Cirrus, SWIR 1 and SWIR 2 bands were used. The characteristics of the Sentinel 2 Level 2A image are given in Table 1 (ESA Sentinel-2 User Handbook, 2015).

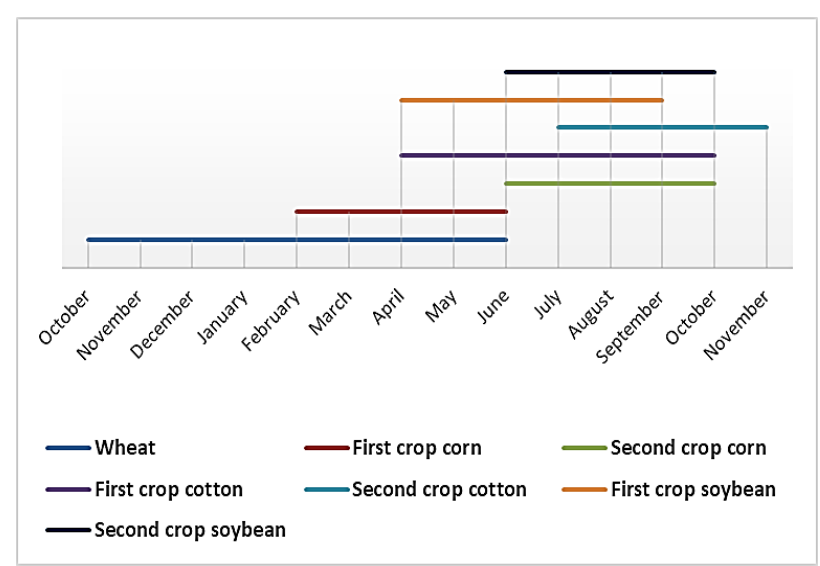

Figure 2. Cultivation timetable for major crops in Çukurova.

\begin{tabular}{|l|l|}
\hline \multicolumn{2}{|c|}{ SENTINEL 2 } \\
\hline Total Spectral Bands & 13 \\
\hline Spectral Resolution (nm) & 433 - 2280 \\
\hline \multirow{3}{*}{ Spatial Resolution (m) } & B2, B3, B4, B8: 10 \\
\cline { 2 - 2 } & B5, B6, B7, B8A, B11, B12: 20 \\
\cline { 2 - 2 } & B1, B9, B10: 60 \\
\hline Radiometric Resolution (bit) & 12 \\
\hline Temporal Resolution (days) & 5 \\
\hline
\end{tabular}

Table 1. The characteristics of Sentinel 2 satellite data (ESA Sentinel-2 User Handbook, 2015).

\subsection{Methodology}

The flow chart of the methodology of the study was shown in Figure 3.

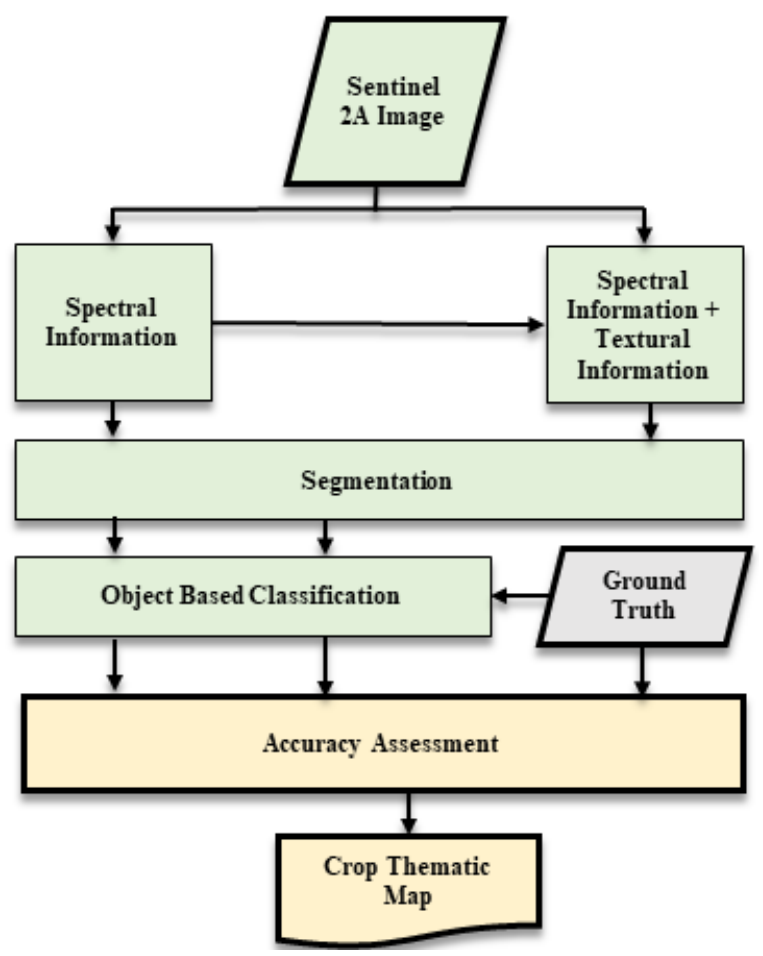

Figure 3. Flowchart of the methodology used. 
As seen in Figure 3, not only spectral bands but also textural features were used as input features in the classification process. In this context, GLCM, which is second-order statistics methods based on (local) information about gray levels in pair of pixels, was used to extract textural features. There are wide varieties of textural features in the literature (Peña-Barragán et al., 2011; Kim and Yeom, 2014; Şall1, 2020). It was seen that the homogeneity and dissimilarity suggested in the literature were not suitable textures to increase the classification accuracy, therefore entropy, standard deviation, mean textural features were found to be the most suitable. In Table 2, the characteristics of the GLCM textural features used in this study are given.

It has been shown in some case studies that the integration of spectral and textural features improves the classification accuracy (Kabir et al., 2010; Zhang et al., 2017). The effect of textural features on classification accuracy may depend on different factors such as appropriate kernel size, direction, specific crop types, study area, image type, etc. (Kwak and Park, 2019).

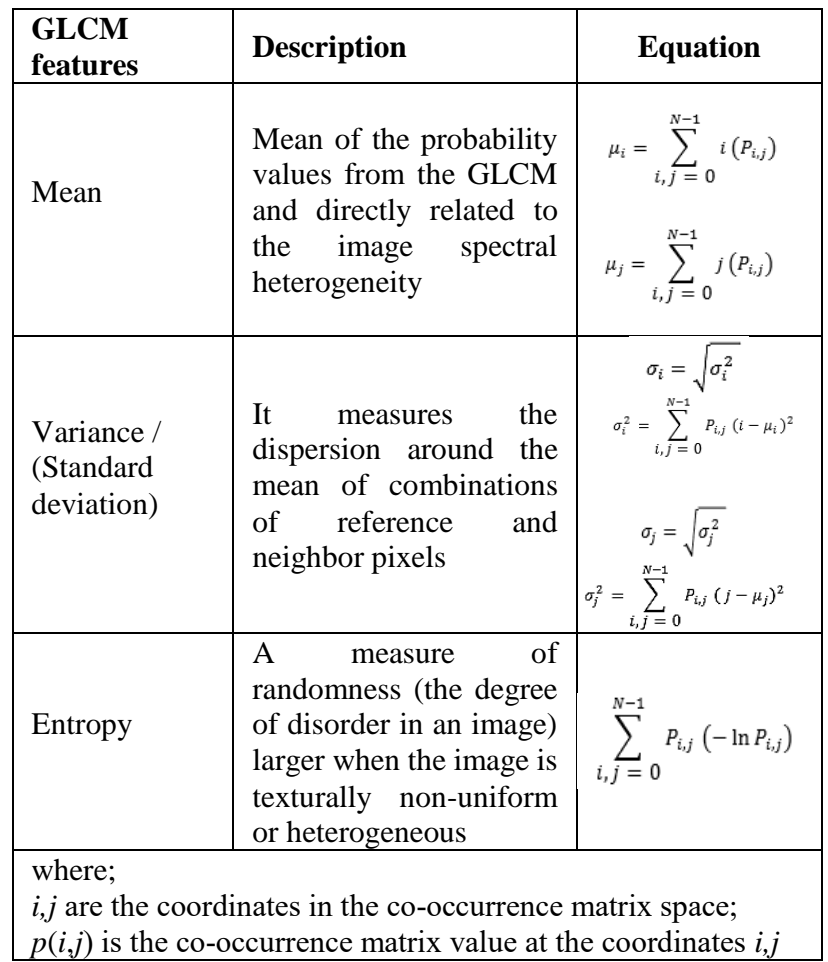

Table 2. The GLCM textural features used in this study (Peña-Barragán et al., 2011; Kwak and Park, 2019).

In the object-based classification method, the segmentation step was applied first to generate the objects. For that purpose, multiresolution segmentation approach was utilized (Baatz and Schäpe, 2000). Homogeneity, color, shape, smoothness and compactness are main criteria that affect segmentation step (Xian, 2015). In this study, using trial and error approach, scale, shape and compactness parameters were determined as $10,0.3$ and 0.6 , respectively, to create meaningful image segments covering agricultural fields. (Mathieu et al., 2007).

In the object-based image classification, the Support Vector Machine (SVM) algorithm, which is widely applied for crop classification in the last decade, was used. SVM is a discriminative classifier defined by a separating hyperplane in $\mathrm{N}$ dimensional space $(\mathrm{N}-$ the number of features) to distinctly classify the data points (Vapnik, 1979, Vapnik, 1999). RBF (Radial Basis Function), which was a nonlinear kernel, was selected as a kernel type. Grid search method was used to determine hyperparameters (i.e. Gamma and c).

Methods similar to those used for pixel-based image classification are also used to verify the results of object-based image classification so that the quality of distribution of segments to object classes is evaluated. Therefore, in this study, Error matrices were created to evaluate the accuracy of classification results.

\section{APPLICATION \& RESULTS}

As a first step, the textural features selected as Mean, Standard deviation and Entropy were generated by considering all directions and the offset was set as 1 in the calculation. The statistical information about textural features is shown in Table 3.

\begin{tabular}{|l|c|c|c|c|c|c|}
\hline \multirow{2}{*}{ Class } & \multicolumn{2}{|c|}{ Entropy } & \multicolumn{2}{c|}{ Std Dev } & \multicolumn{2}{c|}{ Mean } \\
\cline { 2 - 7 } & Min & Max & Min & Max & Min & Max \\
\hline Soybean & 3.52 & 5.67 & 20.21 & 61.40 & 107.59 & 144.17 \\
\hline Corn & 3.86 & 4.85 & 22.68 & 53.84 & 117.83 & 130.42 \\
\hline
\end{tabular}

Table 3. Statistical analysis of the textural features used.

When analysed statistically, it was seen that the interval between minimum and maximum values in entropy is much than the other two textural features. As an example, in a small part of the study area, all the textural features used are shown in Figure 4.

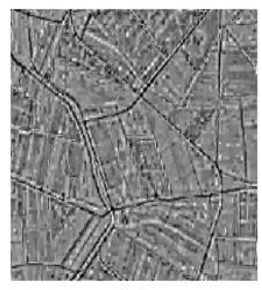

(a)

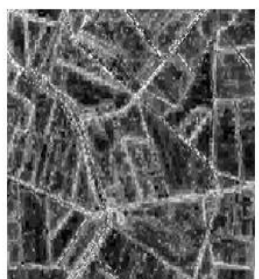

(b)

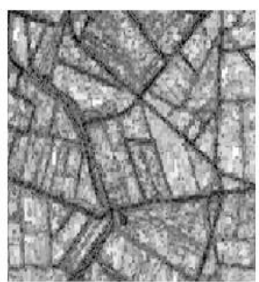

(c)
Figure 4. Example of textural features used a) Mean, b) Standard deviation, c) Entropy.

As seen in Figure 4, apart from spectral heterogeneity, some linear features such as roads, field boundaries, etc. can be easily detected.

The land cover/land use classes considered in the study are corn, soybean, other crops, water, urban and barren land. Multispectral and textural features were used as an input separately and/or in combination to evaluate the potential of texture in differentiating crop types and the accuracy of output thematic maps. In other words, object-based classification was applied to three different datasets; i.e. i) spectral image dataset (original spectral bands - SB) and ii) combination of the spectral and texture image dataset.

Before the classification, multiresolution segmentation approach was applied and the three different parameters, which are scale, shape and compactness, were determined as $10,0.3$ and 0.6 , respectively, using the trial and error approach. After 
segmentation, SVM was applied for different eight combination image dataset.

To visually demonstrate the effect of the texture, the results of only 2 image combinations out of 8 combinations (i.e. using spectral bands only and using spectral bands and all textural features) are shown in Figure 5 and Figure 6, respectively.

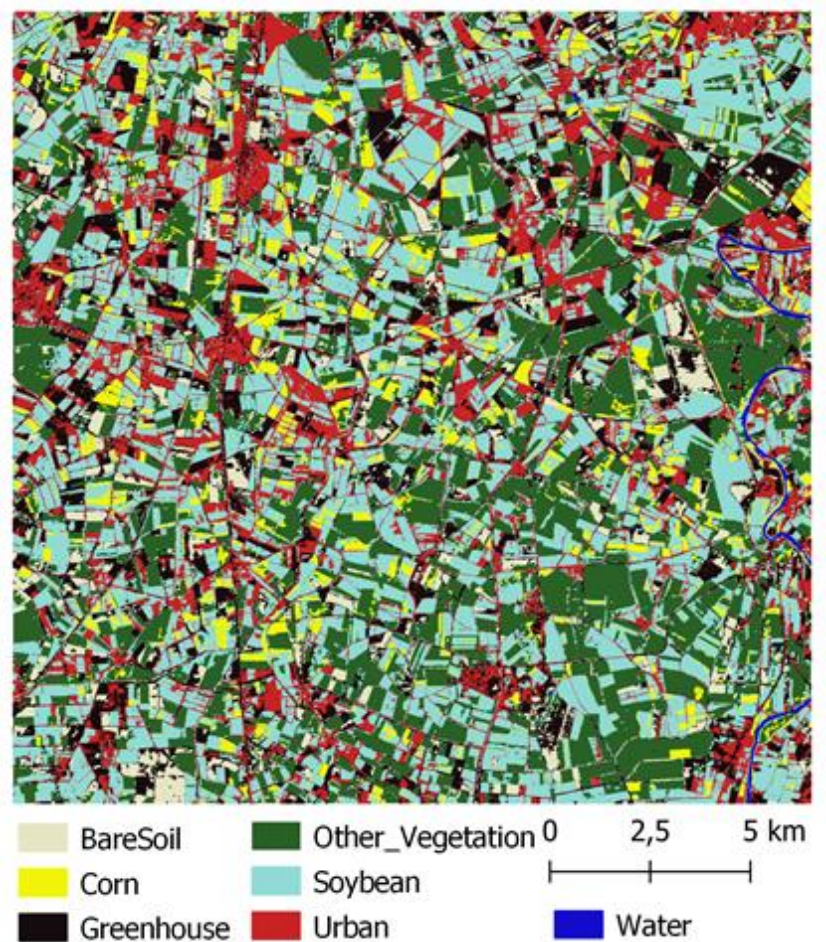

Figure 5. Image classification results conducted with only spectral bands.

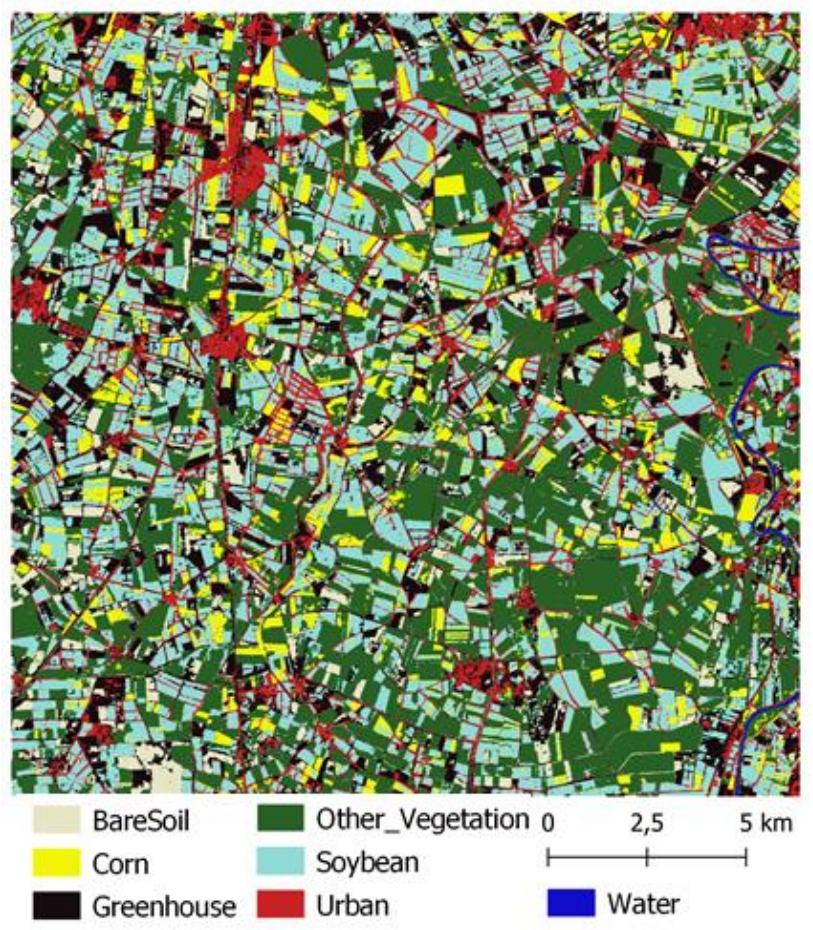

Figure 6. Image classification results conducted with spectral bands \& all 3 textures (entropy, standard deviation, mean).
When the classification results were examined visually, it was seen that the accuracy of the classification increased by adding 3 textural features (i.e. Entropy, Standard deviation, Mean) to the input feature space. First of all, it was observed that linear objects are determined more accurately using textural features. For example, the boundaries of soybean and corn fields are more clearly visible in Figure 6 compared to Figure 5. A similar situation is valid for roads belonging to urban class. In addition, it was determined in Figure 5 that there are some regions that are misclassified as urban class although they belong to greenhouse class. These regions were classified more accurately when the textural features were added to the input feature space as it was seen in Figure 6. In all combinations, water class were classified accurately.

For this purpose, in situ data was used as ground truth data. The comparison of Overall Accuracy and Kappa coefficient for eight different image classification combinations is shown in Figure 7.

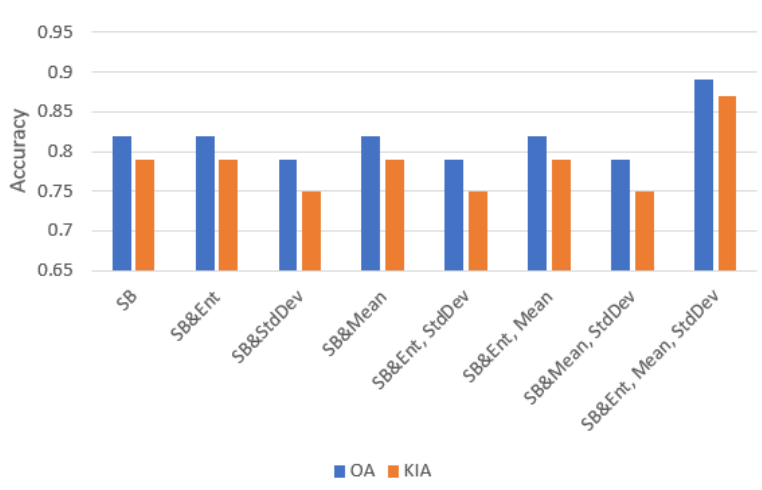

Figure 7. Overall Accuracy and Kappa statistics of classification results with and without texture features.

As shown in Figure 7, the effect of adding only one or two texture features to spectral bands causes slightly increase in accuracy, while the effect of the combination of spectral band and all textures is greater.

As shown in this study (Figure 8) and some studies in the literature (Daughtry, 2001), since the spectral reflectance curves of soybean and corn species have similar spectral reflectance properties, it was clearly seen that additional features such as NDVI, GLCM textures etc. are effective in increasing the classification accuracy.

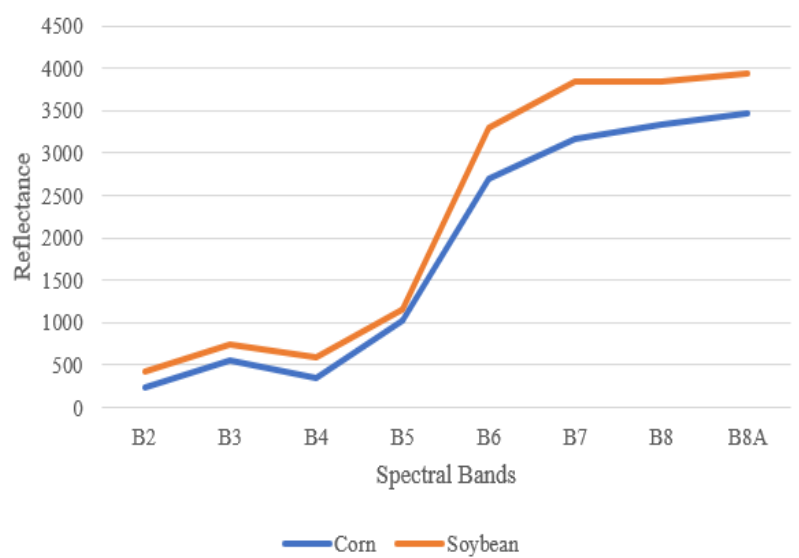

Figure 8. Similar spectral reflectance properties of corn and soybean species. 
The User's Accuracy (UA) and Producer's Accuracy (PA) of all land use/land cover classes obtained from the classification results with and without texture features are given in Figure 9.

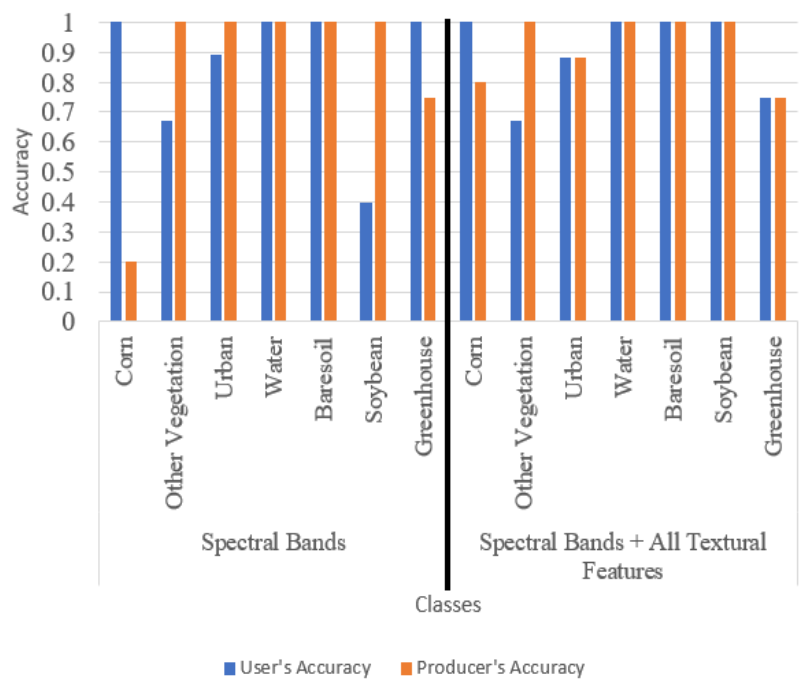

Figure 9. Accuracy metrics of land use/land cover classes obtained from the classification results with and without texture features.

As can be seen in Figure 9, there is an increase in UA and PA metrics of all classes considered. Therefore, this confirms once again the positive effect of using textures in image classification.

\section{CONCLUSION}

In this study, image classification in different combinations (spectral, and spectral \& textural) was performed in order to determine how textural information affects the accuracy of the classification. For this purpose, an object-based classification was performed using eight different feature combinations.

Image classifications made with spectral bands only and spectral bands together with 3 textural features were compared to evaluate the effect of texture on classification accuracy. The Overall Accuracy and Kappa coefficients of these two image classification results were obtained as $0.82 / 0.79$ and $0.89 / 0.87$, respectively. In other words, it was seen that textural features provide $7 \%$ and $8 \%$ increase in Overall Accuracy and Kappa coefficient. When the results were compared visually, it was seen that textural features extracted linear features such as field borders and roads more accurately.

This study is a preliminary study to evaluate the effect of the textural features on crop classification. As a future study, other several parameters (i.e. direction, kernel size, image type and band combinations, different classifiers) that affect the accuracy of the classification will be considered. In addition, it is planned to investigate the effect of using high spatial resolution images and/or parcel-based classification on the identification and mapping of different crop types in the area.

\section{REFERENCES}

Akkartal, A., Türüdü, O., Sunar Erbek, F., 2004: Analysis of changes in vegetation biomass using multi-temporal and multisensor satellite data." In XXXV ISPRS Congress, pp. 12-23. 2004.

Alphan, H., Yilmaz, K.T., 2005: Monitoring Environmental Changes in the Mediterranean Coastal Landscape: The Case of Çukurova, Turkey. Environmental Management, 35(5), 607619. https://doi.org/10.1007/s00267-004-0222-7

Avci, Z.D.U., Sunar, F., 2015: Process-based image analysis for agricultural mapping: A case study in Turkgeldi region, Turkey. Advances in Space Research, 56(8), pp.1635-1644.

Baatz, M., Schäpe, A., 2000: Multiresolution Segmentation: an optimization approach for high quality multi-scale image segmentation, In: Strobl, J., Blaschke, T. and Griesbner, G., Eds., Angewandte Geographische Informations-Verarbeitung, XII, Wichmann Verlag, Karlsruhe, Germany, 12, pp.12-23.

Blaschke, T., 2010: Object based image analysis for remote sensing. ISPRS Journal of Photogrammetry and Remote Sensing, 65(1), 2-16.

Daughtry, C. S., 2001: Discriminating crop residues from soil by shortwave infrared reflectance. Agronomy Journal, 93(1), 125131.

ESA Sentinel-2 User Handbook, 2015: User Handbook. ESA Standard Document.

Goodin, D. G., Anibas, K. L., Bezymennyi, M., 2015: Mapping land cover and land use from object-based classification: an example from a complex agricultural landscape. International Journal of Remote Sensing, 36(18), 4702-4723.

Haralick, R. M., Shanmugam, K., Dinstein, I., 1973: Textural features for image classification. IEEE Transactions on Systems, Man, and Cybernetics, SMC-3(6), 610-621.

Kabir, S., He, D. C., Sanusi, M. A., Wan Hussina, W. M. A., 2010: Texture analysis of IKONOS satellite imagery for urban land use and land cover classification. The Imaging Science Journal, 58(3), 163-170.

Kim, H. O., Yeom, J. M., 2014: Effect of red-edge and texture features for object-based paddy rice crop classification using RapidEye multi-spectral satellite image data. International Journal of Remote Sensing, 35(19), 7046-7068.

Kwak, G. H., Park, N.W., 2019: Impact of texture information on crop classification with machine learning and UAV images. Applied Sciences, 9(4), 643.

Mathieu, R., Aryal, J., Chong, A. K., 2007: Object-based classification of Ikonos imagery for mapping large-scale vegetation communities in urban areas. Sensors, 7(11), 28602880 .

Ok, A., Akar, O., Gungor, O., 2012: Evaluation of random forest method for agricultural crop classification. European Journal of Remote Sensing, 45(1), 421-432. 
Peña-Barragán, J. M., Ngugi, M. K., Plant, R. E., Six, J., 2011: Object-based crop identification using multiple vegetation indices, textural features and crop phenology. Remote Sensing of Environment, 115(6), 1301-1316.

Şall1, B., 2020: Random Forest Classification of Tomato Fields with Planet Satellite Image Data and Accuracy Assessment, MSc Thesis, ITU Graduate School of Science Engineering and Technology.

Trias-Sanz, R., Stamon, G., Louchet, J., 2008: Using Colour, Texture, and Hierarchial Segmentation for High-Resolution Remote Sensing. ISPRS Journal of Photogrammetry and Remote Sensing, 63 (2): 156-168. doi:10.1016/j.isprsjprs.2007.08.005.

TURKSTAT, 2020: Agricultural Land [Statistical Tables].

Retrieved from

https://data.tuik.gov.tr/Kategori/GetKategori?p=Tarim-111, retrieved date 30.01.2021.

United Nations, 2019: World Population Prospects 2019: Highlights (ST/ESA/SER.A/423), Department of Economic and Social Affairs, Population Division.

Vapnik, V., 1979: Estimation of dependences Based on Empirical Data. [in Russian]. Nauka, Moscow. (English translation: Springer-Verlag, New York).

Vapnik, V., 1999: The Nature of Statistical Learning Theory. Springer Science \& Business Media, New York.

Xian, G. Z., 2015: Remote sensing applications for the urban environment, pp.16-22, 978-1-4200-8985-1, CRC Press, New York.

Zainal, Z., Ramli, R., Mustafa, M.M., 2013: Grey-level cooccurrence matrix performance evaluation for heading angle estimation of moveable vision system in static environment. Journal of Sensors, 1-6. doi.org/10.1155/2013/624670.

Zhang, X., Cui, J., Wang, W., Lin, C., 2017: A study for texture feature extraction of high-resolution satellite images based on a direction measure and gray level co-occurrence matrix fusion algorithm. Sensors, 17(7), 1474. 